\title{
Momentum space 3N Faddeev calculations of hadronic and electromagnetic reactions with proton-proton Coulomb and three-nucleon forces included
}

\author{
H. Witała ${ }^{1, a}$, R. Skibiński ${ }^{1}$, J. Golak ${ }^{1}$, and W. Glöckle ${ }^{2}$ \\ 1 M. Smoluchowski Institute of Physics, Jagiellonian University, PL-30059 Kraków, Poland \\ ${ }^{2}$ Institut für theoretische Physik II, Ruhr-Universität Bochum, D-44780 Bochum, Germany
}

\author{
Received: 15 December 2010 / Revised: 24 January 2011 \\ Published online: 2 March 2011 \\ (C) The Author(s) 2011. This article is published with open access at Springerlink.com \\ Communicated by M.C. Birse
}

\begin{abstract}
We extend our approach to incorporate the proton-proton (pp) Coulomb force into the threenucleon $(3 \mathrm{~N})$ momentum space Faddeev calculations of elastic proton-deuteron (pd) scattering and breakup to the case when also a three-nucleon force (3NF) is acting. In addition, we formulate that approach in the application to electron- and $\gamma$-induced reactions on ${ }^{3} \mathrm{He}$. The main new ingredient is a 3-dimensional screened pp Coulomb $t$-matrix obtained by a numerical solution of a 3-dimensional Lippmann-Schwinger equation (LSE). The resulting equations have the same structure as the Faddeev equations which describe pd scattering without $3 \mathrm{NF}$ acting. That shows the practical feasibility of both presented formulations.
\end{abstract}

\section{Introduction}

The long-range nature of the Coulomb force prevents the application of the standard techniques developed for short-range interactions in the analysis of nuclear reactions involving two protons. One proposal to avoid the difficulties including the Coulomb force is to use a screened Coulomb interaction and to reach the pure Coulomb limit through application of a renormalisation procedure [1-4].

Elastic pd scattering first calculations, with modern nuclear forces and the exact Coulomb force in coordinate representation included, have been achieved in a variational hyperspherical harmonic approach [5]. Recently, the inclusion of the Coulomb force was undertaken also for the pd breakup reaction using a screened pp Coulomb force in momentum space and in a partial-wave basis [6]. To get the final predictions which can be compared to the data, the limit to the unscreened situation has been performed numerically applying a renormalization to the resulting $3 \mathrm{~N}$ on-shell amplitudes $[6,7]$.

One main concern in such type of calculations is the application of a partial-wave decomposition to the longranged Coulomb force. Even when screening is applied, it seems reasonable to treat from the beginning the screened pp Coulomb t-matrix without partial-wave decomposition because the required limit of vanishing screening leads necessarily to a drastic increase of the number of partial-wave

\footnotetext{
${ }^{a}$ e-mail: witala@if.uj.edu.pl
}

states involved [8]. In consequence, this leads to an explosion of the number of $3 \mathrm{~N}$ partial waves required for convergence. The very successful approach to include the pp Coulomb force into the $3 \mathrm{~N}$ Faddeev calculations of refs. [6] and [7] revealed a fast convergence in the screening radius using a two-nucleon partial-wave basis of large size. It appears that an independent calculational scheme should be carried through where the treatment of the Coulomb part totally avoids a partial-wave decomposition and thus providing an independent check of the results obtained in $[6,7]$.

Therefore, we developed in $[9,10]$ a novel approach to include the pp Coulomb force into the momentum space $3 \mathrm{~N}$ Faddeev calculations. It is based on a standard formulation for short-range forces and relies on the screening of the long-range Coulomb interaction. In order to avoid all uncertainties connected with the application of the partial-wave expansion, inadequate when working with long-range forces, we used directly the 3-dimensional pp screened Coulomb $t$-matrix. We demonstrated in $[9,10]$ the feasibility of that approach in the case of elastic pd scattering and breakup using a simple dynamical model for the nuclear part of the interaction. In this first study, we applied the most simple exponential screening of the Coulomb force with power $n=1$ for which the 3-dimensional momentum space matrix element can be obtained analytically. Approximating the 3-dimensional pp screened Coulomb $t$-matrix by the potential allowed us to avoid the time-consuming, many-dimensional interpo- 
lations when solving the Faddeev equations. In addition, when calculating the observables, we neglected in the transition amplitude the last term of eq. (62) of ref. [9]. However, in future applications to data analysis and particularily when the comparison to the approach of ref. [7] will be performed, both these approximations must be removed.

In the present paper we extend that approach to include a $3 \mathrm{NF}$ into that formulation. Also, we show how that formulation can be applied to electromagnetic processes induced by electrons or $\gamma$ 's on ${ }^{3} \mathrm{He}$.

In sect. 2, for the convenience of the reader, we shortly describe the main points of the formalism outlined in detail in $[9,10]$ for the case of $3 \mathrm{~N}$ Faddeev calculations with pairwise forces only and extend the corresponding equations to the case when a $3 \mathrm{NF}$ is also acting. In sect. 3 we apply that formulation to electromagnetic reactions on ${ }^{3}$ He. The summary is given in sect. 4 .

\section{Faddeev equations with screened pp Coulomb force}

When only pairwise forces are acting we use the Faddeev equation in the form $[11,12]$

$$
T|\Phi\rangle=t P|\Phi\rangle+t P G_{0} T|\Phi\rangle,
$$

where the permutation operator $P$ is defined in terms of transposition operators $P_{i j}$ of nucleons $i$ and $j, P=$ $P_{12} P_{23}+P_{13} P_{23}, G_{0}$ is the free $3 \mathrm{~N}$ propagator, and $|\Phi\rangle$ is the initial state composed of a deuteron state and a momentum eigenstate of the proton. Knowing $T|\Phi\rangle$ the breakup as well as the elastic pd scattering amplitudes can be gained in the standard manner [11]. The physical content of eq. (1) is revealed after iterating it. The resulting multiple-scattering series contains all possible rescattering contributions induced by interactions of three nucleons and free propagation in between.

We use our standard momentum space partial-wave basis $|p q \tilde{\alpha}\rangle$

$$
|p q \tilde{\alpha}\rangle \equiv\left|p q(l s) j\left(\lambda \frac{1}{2}\right) I(j I) J\left(t \frac{1}{2}\right) T\right\rangle
$$

and distinguish between the partial-wave states $|p q \alpha\rangle$ and $|p q \beta\rangle$. The $|p q \alpha\rangle$ are states with total $2 \mathrm{~N}$ angular momentum $j$ below some value $j_{\max }: j \leq j_{\max }$, in which the nuclear, $V_{N}$, as well as the pp screened Coulomb interaction, $V_{c}^{R}$ (in isospin $t=1$ states only), are acting. In the states $|p q \beta\rangle$ with $j>j_{\max }$, only $V_{c}^{R}$ is acting in the pp subsystem. The states $|p q \alpha\rangle$ and $|p q \beta\rangle$ form a complete set of states

$$
\begin{aligned}
& \int p^{2} \mathrm{~d} p q^{2} \mathrm{~d} q \sum_{\tilde{\alpha}}|p q \tilde{\alpha}\rangle\langle p q \tilde{\alpha}|= \\
& \int p^{2} \mathrm{~d} p q^{2} \mathrm{~d} q\left(\sum_{\alpha}|p q \alpha\rangle\left\langle p q \alpha\left|+\sum_{\beta}\right| p q \beta\right\rangle\langle p q \beta|\right)=\mathrm{I} .
\end{aligned}
$$

Projecting eq. (1) for $T|\Phi\rangle$ on the $|p q \alpha\rangle$ and $|p q \beta\rangle$ states one gets the following system of coupled integral equations:

$$
\begin{aligned}
& \langle p q \alpha|T| \Phi\rangle=\left\langle p q \alpha\left|t_{N+c}^{R} P\right| \Phi\right\rangle \\
& +\left\langle p q \alpha\left|t_{N+c}^{R} P G_{0} \sum_{\alpha^{\prime}} \int p^{\prime 2} \mathrm{~d} p^{\prime} q^{\prime 2} \mathrm{~d} q^{\prime}\right| p^{\prime} q^{\prime} \alpha^{\prime}\right\rangle\left\langle p^{\prime} q^{\prime} \alpha^{\prime}|T| \Phi\right\rangle \\
& +\left\langle p q \alpha\left|t_{N+c}^{R} P G_{0} \sum_{\beta^{\prime}} \int p^{\prime 2} \mathrm{~d} p^{\prime} q^{\prime 2} \mathrm{~d} q^{\prime}\right| p^{\prime} q^{\prime} \beta^{\prime}\right\rangle\left\langle p^{\prime} q^{\prime} \beta^{\prime}|T| \Phi\right\rangle \\
& \langle p q \beta|T| \Phi\rangle=\left\langle p q \beta\left|t_{c}^{R} P\right| \Phi\right\rangle \\
& +\left\langle p q \beta\left|t_{c}^{R} P G_{0} \sum_{\alpha^{\prime}} \int p^{\prime 2} \mathrm{~d} p^{\prime} q^{\prime 2} \mathrm{~d} q^{\prime}\right| p^{\prime} q^{\prime} \alpha^{\prime}\right\rangle\left\langle p^{\prime} q^{\prime} \alpha^{\prime}|T| \Phi\right\rangle \\
& +\left\langle p q \beta\left|t_{c}^{R} P G_{0} \sum_{\beta^{\prime}} \int p^{\prime 2} \mathrm{~d} p^{\prime} q^{\prime 2} \mathrm{~d} q^{\prime}\right| p^{\prime} q^{\prime} \beta^{\prime}\right\rangle\left\langle p^{\prime} q^{\prime} \beta^{\prime}|T| \Phi\right\rangle
\end{aligned}
$$

where $t_{N+c}^{R}$ and $t_{c}^{R}$ are $t$-matrices generated through a LSE by the interactions $V_{N}+V_{c}^{R}$ and $V_{c}^{R}$, respectively. Namely, for states $|\alpha\rangle$ with a two-nucleon subsystem total isospin $t=1$ the corresponding $t$-matrix element $\langle p \alpha| t_{N+c}^{R}(E-$ $\left.\frac{3}{4 m} q^{2}\right)\left|p^{\prime} \alpha^{\prime}\right\rangle$ is a linear combination of the pp, $t_{p p+c}^{R}$, and the neutron-proton (np), $t_{n p}, t=1 t$-matrices, which are generated by the interactions $V_{p p}^{\text {strong }}+V_{c}^{R}$ and $V_{n p}^{\text {strong }}$, respectively. The coefficients of that combination depend on the total $3 \mathrm{~N}$ isospin $T$ and $T^{\prime}$ of the states $|\alpha\rangle$ and $\left|\alpha^{\prime}\right\rangle[9,13]$ :

$$
\begin{aligned}
& \left\langle t=1 T=\frac{1}{2}\left|t_{N+c}^{R}\right| t^{\prime}=1 T^{\prime}=\frac{1}{2}\right\rangle=\frac{1}{3} t_{n p}+\frac{2}{3} t_{p p+c}^{R} \\
& \left\langle t=1 T=\frac{3}{2}\left|t_{N+c}^{R}\right| t^{\prime}=1 T^{\prime}=\frac{3}{2}\right\rangle=\frac{2}{3} t_{n p}+\frac{1}{3} t_{p p+c}^{R} \\
& \left\langle t=1 T=\frac{1}{2}\left|t_{N+c}^{R}\right| t^{\prime}=1 T^{\prime}=\frac{3}{2}\right\rangle=\frac{\sqrt{2}}{3}\left(t_{n p}-t_{p p+c}^{R}\right) \\
& \left\langle t=1 T=\frac{3}{2}\left|t_{N+c}^{R}\right| t^{\prime}=1 T^{\prime}=\frac{1}{2}\right\rangle=\frac{\sqrt{2}}{3}\left(t_{n p}-t_{p p+c}^{R}\right)
\end{aligned}
$$

For the isospin $t=0$, in which case $T=T^{\prime}=\frac{1}{2}$

$$
\left\langle t=0 T=\frac{1}{2}\left|t_{N+c}^{R}\right| t^{\prime}=0 T^{\prime}=\frac{1}{2}\right\rangle=t_{n p} .
$$

In the case of $t_{c}^{R}$ only the screened pp Coulomb force $V_{c}^{R}$ is acting.

The third term on the right-hand side of (5) is proportional to $\left\langle p q \beta\left|t_{c}^{R} P G_{0}\right| p^{\prime} q^{\prime} \beta^{\prime}\right\rangle\left\langle p^{\prime} q^{\prime} \beta^{\prime}\right| t_{c}^{R}$. A direct calculation of its isospin part shows that independently of the value of the total isospin $T$ it vanishes [9].

Inserting $\langle p q \beta|T| \Phi\rangle$ from (5) into (4) one gets

$$
\begin{aligned}
& \langle p q \alpha|T| \Phi\rangle=\left\langle p q \alpha\left|t_{N+c}^{R} P\right| \Phi\right\rangle+\left\langle p q \alpha\left|t_{N+c}^{R} P G_{0} t_{c}^{R} P\right| \Phi\right\rangle \\
& -\left\langle p q \alpha\left|t_{N+c}^{R} P G_{0} \sum_{\alpha^{\prime}} \int p^{\prime 2} \mathrm{~d} p^{\prime} q^{\prime 2} \mathrm{~d} q^{\prime}\right| p^{\prime} q^{\prime} \alpha^{\prime}\right\rangle\left\langle p^{\prime} q^{\prime} \alpha^{\prime}\left|t_{c}^{R} P\right| \Phi\right\rangle
\end{aligned}
$$




$$
\begin{aligned}
& +\left\langle p q \alpha\left|t_{N+c}^{R} P G_{0} \sum_{\alpha^{\prime}} \int p^{\prime 2} \mathrm{~d} p^{\prime} q^{\prime 2} \mathrm{~d} q^{\prime}\right| p^{\prime} q^{\prime} \alpha^{\prime}\right\rangle\left\langle p^{\prime} q^{\prime} \alpha^{\prime}|T| \Phi\right\rangle \\
& +\left\langle p q \alpha\left|t_{N+c}^{R} P G_{0} t_{c}^{R} P G_{0} \sum_{\alpha^{\prime}} \int p^{\prime 2} \mathrm{~d} p^{\prime} q^{\prime 2} \mathrm{~d} q^{\prime}\right| p^{\prime} q^{\prime} \alpha^{\prime}\right\rangle\left\langle p^{\prime} q^{\prime} \alpha^{\prime}|T| \Phi\right\rangle \\
& -\left\langle p q \alpha\left|t_{N+c}^{R} P G_{0} \sum_{\alpha^{\prime}} \int p^{\prime 2} \mathrm{~d} p^{\prime} q^{\prime 2} \mathrm{~d} q^{\prime}\right| p^{\prime} q^{\prime} \alpha^{\prime}\right\rangle\left\langle p^{\prime} q^{\prime} \alpha^{\prime}\right| t_{c}^{R} P G_{0} \\
& \times \sum_{\alpha^{\prime \prime}} \int p^{\prime \prime 2} \mathrm{~d} p^{\prime \prime} q^{\prime \prime 2} \mathrm{~d} q^{\prime \prime}\left|p^{\prime \prime} q^{\prime \prime} \alpha^{\prime \prime}\right\rangle\left\langle p^{\prime \prime} q^{\prime \prime} \alpha^{\prime \prime}|T| \Phi\right\rangle .
\end{aligned}
$$

This is a coupled set of integral equations in the space of the states $|\alpha\rangle$ only, which incorporates the contributions of the pp Coulomb interaction from all partial-wave states up to infinity. It can be solved by iteration and Pade summation $[9,11]$.

When compared to our standard treatment without screened Coulomb force [11] there are two new leading terms: $\left\langle p q \alpha\left|t_{N+c}^{R} P G_{0} t_{c}^{R} P\right| \Phi\right\rangle$ and $-\left\langle p q \alpha\left|t_{N+c}^{R} P G_{0}\right| \alpha^{\prime}\right\rangle \times$ $\left\langle\alpha^{\prime}\left|t_{c}^{R} P\right| \Phi\right\rangle$. The first term must be calculated using directly the 3 -dimensional screened Coulomb $t$-matrix $t_{c}^{R}$, while the second term requires only the partial-wave projected screened Coulomb $t$-matrix elements in the $|\alpha\rangle$ channels. The kernel also contains two new terms: the term $\left\langle p q \alpha\left|t_{N+c}^{R} P G_{0} t_{c}^{R} P G_{0}\right| \alpha^{\prime}\right\rangle\left\langle\alpha^{\prime}|T| \Phi\right\rangle$ must again be calculated with a 3 -dimensional screened Coulomb $t$ matrix $t_{c}^{R}$, while the second one, $-\left\langle p q \alpha\left|t_{N+c}^{R} P G_{0}\right| \alpha^{\prime}\right\rangle \times$ $\left\langle\alpha^{\prime}\left|t_{c}^{R} P G_{0}\right| \alpha^{\prime \prime}\right\rangle\left\langle\alpha^{\prime \prime}|T| \Phi\right\rangle$, involves only the partial-waveprojected screened Coulomb $t$-matrix elements in the $|\alpha\rangle$ channels. The calculation of those new terms with the partial-wave-projected Coulomb $t$-matrices follows our standard procedure [11]. Namely, the two sub-kernels $t_{N+c}^{R} P G_{0}$ and $t_{c}^{R} P G_{0}$ are applied consecutively to the corresponding state. The detailed expressions how to calculate the new terms with the 3-dimensional screened Coulomb $t$-matrix are given in appendix A of ref. [9].

The transition amplitude for breakup, $\left\langle\Phi_{0}\left|U_{0}\right| \Phi\right\rangle$, is given in terms of $T|\Phi\rangle$ by $[11,12]$

$$
\left\langle\Phi_{0}\left|U_{0}\right| \Phi\right\rangle=\left\langle\Phi_{0}|(1+P) T| \Phi\right\rangle,
$$

where $\left|\Phi_{0}\right\rangle \equiv\left|\vec{p} \vec{q} m_{1} m_{2} m_{3} \nu_{1} \nu_{2} \nu_{3}\right\rangle$ is the free state and the Jacobi momenta $\vec{p}$ and $\vec{q}$ specify completely a particular exclusive breakup configuration of three outgoing nucleons. The permutations acting in momentum, spin-, and isospin-spaces can be applied to the bra-state $\left\langle\Phi_{0}\right|=\left\langle\vec{p} \vec{q} m_{1} m_{2} m_{3} \nu_{1} \nu_{2} \nu_{3}\right|$ changing the sequence of nucleons spin and isospin magnetic quantum numbers $m_{i}$ and $\nu_{i}$ and leading to well-known linear combinations of the Jacobi momenta $\vec{p}$ and $\vec{q}$. Thus evaluating (9), it is sufficient to regard the general amplitudes $\left\langle\vec{p} \vec{q} m_{1} m_{2} m_{3} \nu_{1} \nu_{2} \nu_{3}|T| \Phi\right\rangle \equiv\langle\vec{p} \vec{q}|T| \Phi\rangle$. Using eq. (5) and the completeness relation (3) one gets:

$$
\begin{gathered}
\langle\vec{p} q|T| \Phi\rangle=\left\langle\vec{p} \vec{q}\left|\sum_{\alpha^{\prime}} \int p^{\prime 2} \mathrm{~d} p^{\prime} q^{\prime 2} \mathrm{~d} q^{\prime}\right| p^{\prime} q^{\prime} \alpha^{\prime}\right\rangle\left\langle p^{\prime} q^{\prime} \alpha^{\prime}|T| \Phi\right\rangle \\
-\left\langle\vec{p} \vec{q}\left|\sum_{\alpha^{\prime}} \int p^{\prime 2} \mathrm{~d} p^{\prime} q^{\prime 2} \mathrm{~d} q^{\prime}\right| p^{\prime} q^{\prime} \alpha^{\prime}\right\rangle\left\langle p^{\prime} q^{\prime} \alpha^{\prime}\left|t_{c}^{R} P\right| \Phi\right\rangle
\end{gathered}
$$

$$
\begin{aligned}
& -\left\langle\vec{p} \vec{q}\left|\sum_{\alpha^{\prime}} \int p^{\prime 2} \mathrm{~d} p^{\prime} q^{\prime 2} \mathrm{~d} q^{\prime}\right| p^{\prime} q^{\prime} \alpha^{\prime}\right\rangle\left\langle p^{\prime} q^{\prime} \alpha^{\prime}\right| t_{c}^{R} P G_{0} \\
& \times \sum_{\alpha^{\prime \prime}} \int p^{\prime 2} \mathrm{~d} p^{\prime \prime} q^{\prime \prime 2} \mathrm{~d} q^{\prime \prime}\left|p^{\prime \prime} q^{\prime \prime} \alpha^{\prime \prime}\right\rangle\left\langle p^{\prime \prime} q^{\prime \prime} \alpha^{\prime \prime}|T| \Phi\right\rangle \\
& +\left\langle\vec{p} \vec{q}\left|t_{c}^{R} P\right| \Phi\right\rangle+\langle\vec{p} \vec{q}| t_{c}^{R} P G_{0} \\
& \times \sum_{\alpha^{\prime}} \int p^{\prime 2} \mathrm{~d} p^{\prime} q^{\prime 2} \mathrm{~d} q^{\prime}\left|p^{\prime} q^{\prime} \alpha^{\prime}\right\rangle\left\langle p^{\prime} q^{\prime} \alpha^{\prime}|T| \Phi\right\rangle .
\end{aligned}
$$

It follows that, in addition to the amplitudes $\langle p q \alpha|T| \Phi\rangle$, also the partial-wave-projected amplitudes $\left\langle p q \alpha\left|t_{c}^{R} P\right| \Phi\right\rangle$ and $\left\langle p q \alpha\left|t_{c}^{R} P G_{0}\right| \alpha^{\prime}\right\rangle\left\langle\alpha^{\prime}|T| \Phi\right\rangle$ are required. The expressions for the contributions of these three terms to the transition amplitude for the breakup reaction are given in appendix B of ref. [9].

The last two terms in (10) again must be calculated using directly the 3-dimensional screened Coulomb $t$-matrices. In appendix $\mathrm{C}$ of ref. [9] the expression for $\left\langle\vec{p} \vec{q}\left|t_{c}^{R} P\right| \Phi\right\rangle$ is given and in appendix $\mathrm{D}$ for the last matrix element $\left\langle\vec{p} \vec{q}\left|t_{c}^{R} P G_{0}\right| \alpha^{\prime}\right\rangle\left\langle\alpha^{\prime}|T| \Phi\right\rangle$.

The transition amplitude for elastic scattering, $U$, contains in addition to all rescatterings $P T$ also a direct exchange term $P G_{0}^{-1}$ and is given by

$$
\left\langle\Phi^{\prime}|U| \Phi\right\rangle=\left\langle\Phi^{\prime}\left|P G_{0}^{-1}+P T\right| \Phi\right\rangle,
$$

where the outgoing proton-deuteron state $\left|\Phi^{\prime}\right\rangle$ differs from $|\Phi\rangle$ by the direction of the relative proton-deuteron momentum. It can be obtained by quadrature using (10).

It was shown in [9] that the elastic pd scattering amplitude has a well-defined screening limit and does not require renormalisation. To get the physical breakup amplitude, however, it is unavoidable to perform the renormalisation of the pp half-shell $t$-matrices [10]. Such a behavior of the elastic scattering amplitude in our scheme is in contradiction to that in the screening and renormalization method derived in $[1,2]$ and applied in $[6,7]$. There the screening of the elastic scattering amplitude is unavoidable as was demonstrated in [14]. Very probably it reflects different forms of the equations to be solved. Nevertheless it must be checked if the approximations used in our feasibility calculation of ref. [9], mentioned in the introduction, does not introduce a wrong behavior. The resolution of that contradiction awaits to be solved.

When on top of pairwise forces three nucleons interact also through a $3 \mathrm{NF}$ additional rescatterings generated by that $3 \mathrm{NF}$ appear in the multiple-scattering series and the Faddeev equation for the state $T|\Phi\rangle$ changes to [15]:

$$
\begin{aligned}
T|\Phi\rangle= & t P|\Phi\rangle+\left(1+t G_{0}\right) V_{4}^{(1)}(1+P)|\Phi\rangle+t P G_{0} T|\Phi\rangle \\
& +\left(1+t G_{0}\right) V_{4}^{(1)}(1+P) G_{0} T|\Phi\rangle .
\end{aligned}
$$

The $3 \mathrm{~N}$ force $V_{4}$ is naturaly split into 3 parts

$$
V_{4}=V_{4}^{(1)}+V_{4}^{(2)}+V_{4}^{(3)}
$$

where $V_{4}^{(i)}$ is symmetrical under the exchange of nucleons $j$ and $k(i, j, k=1,2,3, i \neq j \neq k)$. Such a splitting 
is always possible and in the case of the $\pi-\pi$ exchange $3 \mathrm{NF}$ corresponds to the three possible choices of the nucleon undergoing off-shell $\pi \mathrm{N}$ scattering. Equation (12) contains two new terms: one leading term and one in the kernel. They reflect additional contributions to the multiple-scattering series caused by the $3 \mathrm{NF}$.

Performing analogous steps as for (1) and starting with the projection of (12) on the $|p q \alpha\rangle$ and $|p q \beta\rangle$ states one gets

$$
\begin{aligned}
& \langle p q \alpha|T| \Phi\rangle=\left\langle p q \alpha\left|t_{N+c}^{R} P\right| \Phi\right\rangle \\
& \quad+\left\langle p q \alpha\left|\left(1+t_{N+c}^{R} G_{0}\right) V_{4}^{(1)}(1+P)\right| \Phi\right\rangle \\
& \quad+\left\langle p q \alpha\left|t_{N+c}^{R} P G_{0}\right| \alpha^{\prime}\right\rangle\left\langle\alpha^{\prime}|T| \Phi\right\rangle \\
& \quad+\left\langle p q \alpha\left|t_{N+c}^{R} P G_{0}\right| \beta^{\prime}\right\rangle\left\langle\beta^{\prime}|T| \Phi\right\rangle \\
& \quad+\left\langle p q \alpha\left|\left(1+t_{N+c}^{R} G_{0}\right) V_{4}^{(1)}(1+P) G_{0}\right| \alpha^{\prime}\right\rangle\left\langle\alpha^{\prime}|T| \Phi\right\rangle \\
& \quad+\left\langle p q \alpha\left|\left(1+t_{N+c}^{R} G_{0}\right) V_{4}^{(1)}(1+P) G_{0}\right| \beta^{\prime}\right\rangle\left\langle\beta^{\prime}|T| \Phi\right\rangle,
\end{aligned}
$$

and

$$
\begin{aligned}
& \langle p q \beta|T| \Phi\rangle=\left\langle p q \beta\left|t_{c}^{R} P\right| \Phi\right\rangle \\
& +\left\langle p q \beta\left|\left(1+t_{c}^{R} G_{0}\right) V_{4}^{(1)}(1+P)\right| \Phi\right\rangle \\
& +\left\langle p q \beta\left|t_{c}^{R} P G_{0}\right| \alpha^{\prime}\right\rangle\left\langle\alpha^{\prime}|T| \Phi\right\rangle+\left\langle p q \beta\left|t_{c}^{R} P G_{0}\right| \beta^{\prime}\right\rangle\left\langle\beta^{\prime}|T| \Phi\right\rangle \\
& +\left\langle p q \beta\left|\left(1+t_{c}^{R} G_{0}\right) V_{4}^{(1)}(1+P) G_{0}\right| \alpha^{\prime}\right\rangle\left\langle\alpha^{\prime}|T| \Phi\right\rangle \\
& +\left\langle p q \beta\left|\left(1+t_{c}^{R} G_{0}\right) V_{4}^{(1)}(1+P) G_{0}\right| \beta^{\prime}\right\rangle\left\langle\beta^{\prime}|T| \Phi\right\rangle
\end{aligned}
$$

Here and in the following we shortened our notation by neglecting the summation sign over intermediate states $\left|\alpha^{\prime}\right\rangle$ $\left(\left|\beta^{\prime}\right\rangle\right)$ and the integration sign over the corresponding Jacobi momenta $p^{\prime}$ and $q^{\prime}$. Therefore whenever a projection operator $\left|\alpha^{\prime}\right\rangle\left\langle\alpha^{\prime}\right|$ appears in intermediate states means that the following summation and integrations must be performed:

$$
\left|\alpha^{\prime}\right\rangle\left\langle\alpha^{\prime}\left|\equiv \sum_{\alpha^{\prime}} \int p^{\prime 2} \mathrm{~d} p^{\prime} q^{\prime 2} \mathrm{~d} q^{\prime}\right| p^{\prime} q^{\prime} \alpha^{\prime}\right\rangle\left\langle p^{\prime} q^{\prime} \alpha^{\prime}\right| .
$$

Since a $3 \mathrm{NF}$ is short-ranged its matrix elements containing $|\beta\rangle$ channels vanish:

$\left\langle\alpha\left|V_{4}^{(1)}(1+P)\right| \beta\right\rangle=\left\langle\beta\left|V_{4}^{(1)}(1+P)\right| \alpha\right\rangle=\left\langle\beta\left|V_{4}^{(1)}(1+P)\right| \beta\right\rangle=0$.

Thus in the $|\beta\rangle$ channels only the pp Coulomb force is acting and therefore (15) reduces to

$$
\begin{aligned}
& \langle p q \beta|T| \Phi\rangle=\left\langle p q \beta\left|t_{c}^{R} P\right| \Phi\right\rangle \\
& +\left\langle p q \beta\left|t_{c}^{R} P G_{0}\right| \alpha^{\prime}\right\rangle\left\langle\alpha^{\prime}|T| \Phi\right\rangle+\left\langle p q \beta\left|t_{c}^{R} P G_{0}\right| \beta^{\prime}\right\rangle\left\langle\beta^{\prime}|T| \Phi\right\rangle= \\
& \left\langle p q \beta\left|t_{c}^{R} P\right| \Phi\right\rangle+\left\langle p q \beta\left|t_{c}^{R} P G_{0}\right| \alpha^{\prime}\right\rangle\left\langle\alpha^{\prime}|T| \Phi\right\rangle .
\end{aligned}
$$

Again we have used the fact that the third term in (18) vanishes (see also the remark after (7)). Also note that $t_{c}^{R}$ is diagonal in the high partial waves and consequently $V_{4}^{(1)}$ does not contribute.
Inserting (18) into (14) one gets

$$
\begin{aligned}
& \langle p q \alpha|T| \Phi\rangle=\left\langle p q \alpha\left|t_{N+c}^{R} P\right| \Phi\right\rangle \\
& +\left\langle p q \alpha\left|\left(1+t_{N+c}^{R} G_{0}\right) V_{4}^{(1)}(1+P)\right| \Phi\right\rangle \\
& +\left\langle p q \alpha\left|t_{N+c}^{R} P G_{0}\right| \alpha^{\prime}\right\rangle\left\langle\alpha^{\prime}|T| \Phi\right\rangle+\left\langle p q \alpha\left|t_{N+c}^{R} P G_{0}\right| \beta^{\prime}\right\rangle \\
& \times\left[\left\langle\beta^{\prime}\left|t_{c}^{R} P\right| \Phi\right\rangle+\left\langle\beta^{\prime}\left|t_{c}^{R} P G_{0}\right| \alpha^{\prime}\right\rangle\left\langle\alpha^{\prime}|T| \Phi\right\rangle\right] \\
& +\left\langle p q \alpha\left|\left(1+t_{N+c}^{R} G_{0}\right) V_{4}^{(1)}(1+P) G_{0}\right| \alpha^{\prime}\right\rangle\left\langle\alpha^{\prime}|T| \Phi\right\rangle \\
& +\left\langle p q \alpha\left|\left(1+t_{N+c}^{R} G_{0}\right) V_{4}^{(1)}(1+P) G_{0}\right| \beta^{\prime}\right\rangle \\
& \times\left[\left\langle\beta^{\prime}\left|t_{c}^{R} P\right| \Phi\right\rangle+\left\langle\beta^{\prime}\left|t_{c}^{R} P G_{0}\right| \alpha^{\prime}\right\rangle\left\langle\alpha^{\prime}|T| \Phi\right\rangle\right] .
\end{aligned}
$$

Thus,

$$
\begin{aligned}
& \langle p q \alpha|T| \Phi\rangle=\left\langle p q \alpha\left|t_{N+c}^{R} P\right| \Phi\right\rangle \\
& +\left\langle p q \alpha\left|\left(1+t_{N+c}^{R} G_{0}\right) V_{4}^{(1)}(1+P)\right| \Phi\right\rangle \\
& +\left\langle p q \alpha\left|t_{N+c}^{R} P G_{0}\right| \beta^{\prime}\right\rangle\left\langle\beta^{\prime}\left|t_{c}^{R} P\right| \Phi\right\rangle \\
& +\left\langle p q \alpha\left|\left(1+t_{N+c}^{R} G_{0}\right) V_{4}^{(1)}(1+P) G_{0}\right| \beta^{\prime}\right\rangle\left\langle\beta^{\prime}\left|t_{c}^{R} P\right| \Phi\right\rangle \\
& +\left\langle p q \alpha\left|t_{N+c}^{R} P G_{0}\right| \alpha^{\prime}\right\rangle\left\langle\alpha^{\prime}|T| \Phi\right\rangle \\
& +\left\langle p q \alpha\left|t_{N+c}^{R} P G_{0}\right| \beta^{\prime}\right\rangle\left\langle\beta^{\prime}\left|t_{c}^{R} P G_{0}\right| \alpha^{\prime \prime}\right\rangle\left\langle\alpha^{\prime \prime}|T| \Phi\right\rangle \\
& +\left\langle p q \alpha\left|\left(1+t_{N+c}^{R} G_{0}\right) V_{4}^{(1)}(1+P) G_{0}\right| \alpha^{\prime}\right\rangle\left\langle\alpha^{\prime}|T| \Phi\right\rangle \\
& +\left\langle p q \alpha\left|\left(1+t_{N+c}^{R} G_{0}\right) V_{4}^{(1)}(1+P) G_{0}\right| \beta^{\prime}\right\rangle \\
& \times\left\langle\beta^{\prime}\left|t_{c}^{R} P G_{0}\right| \alpha^{\prime}\right\rangle\left\langle\alpha^{\prime}|T| \Phi\right\rangle .
\end{aligned}
$$

Due to (17) the term in the fourth line of (20) and the last term of (20) can be dropped. Using the completeness relation (3) for the $|\alpha\rangle$ and $|\beta\rangle$ states one finally is left with the coupled set of integral equations in the space of $|\alpha\rangle$ channels only:

$$
\begin{aligned}
& \langle p q \alpha|T| \Phi\rangle=\left\langle p q \alpha\left|t_{N+c}^{R} P\right| \Phi\right\rangle \\
& +\left\langle p q \alpha\left|\left(1+t_{N+c}^{R} G_{0}\right) V_{4}^{(1)}(1+P)\right| \Phi\right\rangle \\
& +\left\langle p q \alpha\left|t_{N+c}^{R} P G_{0} t_{c}^{R} P\right| \Phi\right\rangle-\left\langle p q \alpha\left|t_{N+c}^{R} P G_{0}\right| \alpha^{\prime}\right\rangle\left\langle\alpha^{\prime}\left|t_{c}^{R} P\right| \Phi\right\rangle \\
& +\left\langle p q \alpha\left|t_{N+c}^{R} P G_{0}\right| \alpha^{\prime}\right\rangle\left\langle\alpha^{\prime}|T| \Phi\right\rangle \\
& +\left\langle p q \alpha\left|t_{N+c}^{R} P G_{0} t_{c}^{R} P G_{0}\right| \alpha^{\prime}\right\rangle\left\langle\alpha^{\prime}|T| \Phi\right\rangle \\
& -\left\langle p q \alpha\left|t_{N+c}^{R} P G_{0}\right| \alpha^{\prime}\right\rangle\left\langle\alpha^{\prime}\left|t_{c}^{R} P G_{0}\right| \alpha^{\prime \prime}\right\rangle\left\langle\alpha^{\prime \prime}|T| \Phi\right\rangle \\
& +\left\langle p q \alpha\left|\left(1+t_{N+c}^{R} G_{0}\right) V_{4}^{(1)}(1+P) G_{0}\right| \alpha^{\prime}\right\rangle\left\langle\alpha^{\prime}|T| \Phi\right\rangle .
\end{aligned}
$$

Comparing it to eq. (8) with 2-body forces only, there is one additional contribution in the leading term, $\left\langle p q \alpha\left|\left(1+t_{N+c}^{R} G_{0}\right) V_{4}^{(1)}(1+P)\right| \Phi\right\rangle$, and one in the kernel, $\left\langle p q \alpha\left|\left(1+t_{N+c}^{R} G_{0}\right) V_{4}^{(1)}(1+P) G_{0}\right| \alpha^{\prime}\right\rangle\left\langle\alpha^{\prime}|T| \Phi\right\rangle$, both containing $V_{4}^{(1)}(1+P)$.

The transition amplitude for breakup is again given by (9) and for elastic scattering two new terms driven by $V_{4}^{(1)}(1+P)$ appear [15]:

$$
\begin{aligned}
\left\langle\Phi^{\prime}|U| \Phi\right\rangle= & \left\langle\Phi^{\prime}\right| P G_{0}^{-1}+P T+V_{4}^{(1)}(1+P) \\
& +V_{4}^{(1)}(1+P) G_{0} T|\Phi\rangle .
\end{aligned}
$$

Since the structure of the set (21) is analogous to the structure of the set (8) describing pd scattering when only 
pairwise forces are acting, it follows that the inclusion of the $3 \mathrm{NF}$ into the Faddeev calculations of pd elastic scattering and breakup reaction requires no new matrix elements and numerical tools beyond those used in $[9,10]$ and $[15]$.

\section{The electromagnetic reactions on ${ }^{3} \mathrm{He}$}

It was shown in [16] that the basic equations describing reactions on ${ }^{3} \mathrm{He}$ induced by photons or electrons have the same structure as the $3 \mathrm{~N}$ continuum Faddeev equations (1) and (12). The new physical ingredient is the photon absorption operator, lets call it $\mathbf{O}[16]$. For a complete breakup of ${ }^{3} \mathrm{He}$ induced by photons the nuclear matrix element $N$, from which all observables can be determined, is given by an auxiliary state $|U\rangle$ which fulfills the Faddeev-type equation:

$$
\begin{aligned}
& |U\rangle=\left[t G_{0}+\frac{1}{2}(P+1) V_{4}^{(1)} G_{0}\left(1+t G_{0}\right)\right](1+P) \mathbf{O}\left|\Psi_{i}\right\rangle \\
& +\left(t G_{0} P+\frac{1}{2}(P+1) V_{4}^{(1)} G_{0}\left(1+t G_{0}\right) P\right)|U\rangle
\end{aligned}
$$

Then,

$$
N=\left\langle\Phi_{0}\left|\left(1+t G_{0}\right)(1+P) \mathbf{O}\right| \Psi_{i}\right\rangle+\left\langle\Phi_{0}\left|\left(1+t G_{0}\right) P\right| U\right\rangle .
$$

Here $\left|\Psi_{i}\right\rangle$ is the initial ${ }^{3} \mathrm{He}$ bound state and $\left|\Phi_{0}\right\rangle$ is the fully antisymmetrized free state of three outgoing nucleons, given in terms of their Jacobi momenta and spin and isospin quantum numbers.

For the pd breakup of ${ }^{3} \mathrm{He}$ the nuclear matrix element is given [16] by

$$
N_{p d}=\left\langle\Phi_{q}|(1+P) \mathbf{O}| \Psi_{i}\right\rangle+\left\langle\Phi_{q}|P| U\right\rangle,
$$

where the final state is determined by the proton-deuteron relative momentum eigenstate $|\vec{q}\rangle$ and the deuteron wave function $\left|\phi_{d}\right\rangle$ :

$$
\left\langle\Phi_{q}\right|=\left\langle\phi_{d}\right|\langle\vec{q}| .
$$

Let us consider first the case without $3 \mathrm{NFs}$ :

$$
|U\rangle=t G_{0}(1+P) \mathbf{O}\left|\Psi_{i}\right\rangle+t G_{0} P|U\rangle .
$$

Projecting eq. (27) on states $|\alpha\rangle$ and $|\beta\rangle$ (in the $|\beta\rangle$ states only the screened Coulomb force $V_{c}^{R}$ is acting) one gets

$\langle p q \alpha \mid U\rangle=\left\langle p q \alpha\left|t_{N+c}^{R} G_{0}(1+P) \mathbf{O}\right| \Psi_{i}\right\rangle+\left\langle p q \alpha\left|t_{N+c}^{R} G_{0} P\right| U\right\rangle$

and

$$
\begin{aligned}
\langle p q \beta \mid U\rangle= & \left\langle p q \beta\left|t_{c}^{R} G_{0}(1+P) \mathbf{O}\right| \Psi_{i}\right\rangle+\left\langle p q \beta\left|t_{c}^{R} G_{0} P\right| U\right\rangle= \\
& \left\langle p q \beta\left|t_{c}^{R} G_{0}(1+P) \mathbf{O}\right| \Psi_{i}\right\rangle+\left\langle p q \beta\left|t_{c}^{R} G_{0} P\right| \alpha^{\prime}\right\rangle\left\langle\alpha^{\prime} \mid U\right\rangle \\
& +\left\langle p q \beta\left|t_{c}^{R} G_{0} P\right| \beta^{\prime}\right\rangle\left\langle\beta^{\prime} \mid U\right\rangle .
\end{aligned}
$$

Since in above equations the photon absorption operator $\mathbf{O}$ comes always with ${ }^{3} \mathrm{He}$ bound state therefore $\left\langle\beta|\mathbf{O}| \Psi_{i}\right\rangle=\left\langle\beta|P \mathbf{O}| \Psi_{i}\right\rangle=0$. Consequently the first term in (29) vanishes. The last term is proportional to $\langle p q \beta|$ $t_{c}^{R} G_{0} P|\beta\rangle\langle\beta| t_{c}^{R}$ and the direct calculation of its isospin part gives zero. Thus eq. (29) reduces to:

$$
\langle p q \beta \mid U\rangle=\left\langle p q \beta\left|t_{c}^{R} G_{0} P\right| \alpha^{\prime}\right\rangle\left\langle\alpha^{\prime} \mid U\right\rangle .
$$

Inserting (30) into (28) one gets:

$$
\begin{aligned}
& \langle p q \alpha \mid U\rangle=\left\langle p q \alpha\left|t_{N+c}^{R} G_{0}(1+P) \mathbf{O}\right| \Psi_{i}\right\rangle \\
& +\left\langle p q \alpha\left|t_{N+c}^{R} G_{0} P\right| \alpha^{\prime}\right\rangle\left\langle\alpha^{\prime} \mid U\right\rangle+\left\langle p q \alpha\left|t_{N+c}^{R} G_{0} P\right| \beta^{\prime}\right\rangle\left\langle\beta^{\prime} \mid U\right\rangle= \\
& \left\langle p q \alpha\left|t_{N+c}^{R} G_{0}(1+P) \mathbf{O}\right| \Psi_{i}\right\rangle+\left\langle p q \alpha\left|t_{N+c}^{R} G_{0} P\right| \alpha^{\prime}\right\rangle\left\langle\alpha^{\prime} \mid U\right\rangle \\
& +\left\langle p q \alpha\left|t_{N+c}^{R} G_{0} P\right| \beta^{\prime}\right\rangle\left\langle\beta^{\prime}\left|t_{c}^{R} G_{0} P\right| \alpha^{\prime}\right\rangle\left\langle\alpha^{\prime} \mid U\right\rangle .
\end{aligned}
$$

Using the completeness relation for the $|\alpha\rangle$ and $|\beta\rangle$ states gives:

$$
\begin{aligned}
\langle p q \alpha \mid U\rangle= & \left\langle p q \alpha\left|t_{N+c}^{R} G_{0}(1+P) \mathbf{O}\right| \Psi_{i}\right\rangle \\
& +\left\langle p q \alpha\left|t_{N+c}^{R} G_{0} P\right| \alpha^{\prime}\right\rangle\left\langle\alpha^{\prime} \mid U\right\rangle \\
& +\left\langle p q \alpha\left|t_{N+c}^{R} G_{0} P t_{c}^{R} G_{0} P\right| \alpha^{\prime}\right\rangle\left\langle\alpha^{\prime} \mid U\right\rangle \\
& -\left\langle p q \alpha\left|t_{N+c}^{R} G_{0} P\right| \alpha^{\prime}\right\rangle\left\langle\alpha^{\prime}\left|t_{c}^{R} G_{0} P\right| \alpha^{\prime \prime}\right\rangle\left\langle\alpha^{\prime \prime} \mid U\right\rangle .
\end{aligned}
$$

When compared with the set resulting from (27) for a neutron-neutron-proton system there are two new terms in the kernel: $\left\langle p q \alpha\left|t_{N+c}^{R} G_{0} P t_{c}^{R} G_{0} P\right| \alpha^{\prime}\right\rangle\left\langle\alpha^{\prime} \mid U\right\rangle$ and $-\left\langle p q \alpha\left|t_{N+c}^{R} G_{0} P\right| \alpha^{\prime}\right\rangle\left\langle\alpha^{\prime}\left|t_{c}^{R} G_{0} P\right| \alpha^{\prime \prime}\right\rangle\left\langle\alpha^{\prime \prime} \mid U\right\rangle$. They are identical to those in (8) for the $3 \mathrm{~N}$ continuum and consequently also their evaluation is the same as for pd scattering. The vanishing of $|\beta\rangle$-components of the $\mathbf{O}\left|\Psi_{i}\right\rangle$-state caused that, instead of three leading terms as in (8), only one leading term appears, which can be calculated in a standard way [16].

Starting from (23) and performing analogous steps when the $3 \mathrm{NF}$ is included gives

$$
\begin{aligned}
& \langle p q \alpha \mid U\rangle=\left\langle p q \alpha\left|t_{N+c}^{R} G_{0}(1+P) \mathbf{O}\right| \Psi_{i}\right\rangle \\
& \quad+\left\langle p q \alpha\left|\frac{1}{2}(P+1) V_{4}^{(1)} G_{0}\left(1+t_{N+c}^{R} G_{0}\right)(1+P) \mathbf{O}\right| \Psi_{i}\right\rangle \\
& \quad+\left\langle p q \alpha\left|t_{N+c}^{R} G_{0} P\right| \alpha^{\prime}\right\rangle\left\langle\alpha^{\prime} \mid U\right\rangle \\
& \quad+\left\langle p q \alpha\left|t_{N+c}^{R} G_{0} P t_{c}^{R} G_{0} P\right| \alpha^{\prime}\right\rangle\left\langle\alpha^{\prime} \mid U\right\rangle \\
& \quad-\left\langle p q \alpha\left|t_{N+c}^{R} G_{0} P\right| \alpha^{\prime}\right\rangle\left\langle\alpha^{\prime}\left|t_{c}^{R} G_{0} P\right| \alpha^{\prime \prime}\right\rangle\left\langle\alpha^{\prime \prime} \mid U\right\rangle \\
& \quad+\left\langle p q \alpha\left|\frac{1}{2}(P+1) V_{4}^{(1)} G_{0}\left(1+t_{N+c}^{R} G_{0}\right) P\right| \alpha^{\prime}\right\rangle\left\langle\alpha^{\prime} \mid U\right\rangle .
\end{aligned}
$$

Thus, adding a $3 \mathrm{NF}$ results in one additional leading term, $\left\langle p q \alpha\left|\frac{1}{2}(P+1) V_{4}^{(1)} G_{0}\left(1+t_{N+c}^{R} G_{0}\right)(1+P) \mathbf{O}\right| \Psi_{i}\right\rangle$, and one additional kernel term, $\langle p q \alpha| \frac{1}{2}(P+1) V_{4}^{(1)} G_{0}(1+$ $\left.t_{N+c}^{R} G_{0}\right) P\left|\alpha^{\prime}\right\rangle\left\langle\alpha^{\prime} \mid U\right\rangle$.

The matrix elements $\langle p q \alpha \mid U\rangle$ provide transition amplitudes for the two- and three-body breakup of ${ }^{3} \mathrm{He}$. Namely, for the two-body breakup of ${ }^{3} \mathrm{He}$ the second term in (25) can be calculated using (30) and the completeness of the $|\alpha\rangle$ and $|\beta\rangle$ states, resulting in:

$$
\begin{aligned}
\left\langle\Phi_{q}|P| U\right\rangle= & \left\langle\Phi_{q}|P| \alpha\right\rangle\langle\alpha \mid U\rangle+\left\langle\Phi_{q}|P| \beta\right\rangle\langle\beta \mid U\rangle= \\
& \left\langle\Phi_{q}|P| \alpha\right\rangle\langle\alpha \mid U\rangle+\left\langle\Phi_{q}\left|P t_{c}^{R} G_{0} P\right| \alpha^{\prime}\right\rangle\left\langle\alpha^{\prime} \mid U\right\rangle \\
& -\left\langle\Phi_{q}|P| \alpha\right\rangle\left\langle\alpha\left|t_{c}^{R} G_{0} P\right| \alpha^{\prime}\right\rangle\left\langle\alpha^{\prime} \mid U\right\rangle .
\end{aligned}
$$


The first and third terms can be obtained from the $|\alpha\rangle$ partial-wave-projected matrix elements using (B.2) of ref. [9]. The second term must be calculated using directly the 3 -dimensional screened Coulomb $t$-matrix $t_{c}^{R}$ according to (D.9) of ref. [9].

For the three-body breakup of ${ }^{3} \mathrm{He}$ the second term in $(24)$ can be calculated in a similar way and is given by

$$
\begin{aligned}
& \left\langle\Phi_{0}\left|\left(1+t G_{0}\right) P\right| U\right\rangle=\left\langle\Phi_{0}|P| \alpha\right\rangle\langle\alpha \mid U\rangle \\
& +\left\langle\Phi_{0}|P| \beta\right\rangle\left\langle\beta\left|t_{c}^{R} G_{0} P\right| \alpha^{\prime}\right\rangle\left\langle\alpha^{\prime} \mid U\right\rangle \\
& +\left\langle\Phi_{0} \mid \alpha\right\rangle\left\langle\alpha\left|t_{N+c}^{R} G_{0} P\right| U\right\rangle+\left\langle\Phi_{0} \mid \beta\right\rangle\left\langle\beta\left|t_{c}^{R} G_{0} P\right| U\right\rangle= \\
& \left\langle\Phi_{0}|P| \alpha\right\rangle\langle\alpha \mid U\rangle+\left\langle\Phi_{0}\left|P t_{c}^{R} G_{0} P\right| \alpha\right\rangle\langle\alpha \mid U\rangle \\
& -\left\langle\Phi_{0}|P| \alpha\right\rangle\left\langle\alpha\left|t_{c}^{R} G_{0} P\right| \alpha^{\prime}\right\rangle\left\langle\alpha^{\prime} \mid U\right\rangle \\
& +\left\langle\Phi_{0} \mid \alpha\right\rangle\left\langle\alpha\left|t_{N+c}^{R} G_{0} P\right| \alpha^{\prime}\right\rangle\left\langle\alpha^{\prime} \mid U\right\rangle \\
& +\left\langle\Phi_{0} \mid \alpha\right\rangle\left\langle\alpha\left|t_{N+c}^{R} G_{0} P t_{c}^{R} G_{0} P\right| \alpha^{\prime}\right\rangle\langle\alpha \mid U\rangle \\
& -\left\langle\Phi_{0} \mid \alpha\right\rangle\left\langle\alpha\left|t_{N+c}^{R} G_{0} P\right| \alpha^{\prime}\right\rangle\left\langle\alpha^{\prime}\left|t_{c}^{R} G_{0} P\right| \alpha^{\prime \prime}\right\rangle\left\langle\alpha^{\prime \prime} \mid U\right\rangle \\
& +\left\langle\Phi_{0}\left|t_{c}^{R} G_{0} P\right| \alpha\right\rangle\langle\alpha \mid U\rangle-\left\langle\Phi_{0} \mid \alpha\right\rangle\left\langle\alpha\left|t_{c}^{R} G_{0} P\right| \alpha^{\prime}\right\rangle\left\langle\alpha^{\prime} \mid U\right\rangle .
\end{aligned}
$$

Here again, the second, fifth and seventh term must be calculated using directly the 3-dimensional screened Coulomb $t$-matrix $t_{c}^{R}$. For the second, $\left\langle\Phi_{0}\left|P t_{c}^{R} G_{0} P\right| \alpha\right\rangle \times$ $\langle\alpha \mid U\rangle$, and seventh, $\left\langle\Phi_{0}\left|t_{c}^{R} G_{0} P\right| \alpha\right\rangle\langle\alpha \mid U\rangle$, term the calculation follows expressions (D.6), (D.7) and (D.8) of ref. [9]. For the fifth matrix element, $\left\langle\Phi_{0} \mid \alpha\right\rangle \times$ $\left\langle\alpha\left|t_{N+c}^{R} G_{0} P t_{c}^{R} G_{0} P\right| \alpha^{\prime}\right\rangle\left\langle\alpha^{\prime} \mid U\right\rangle$, the corresponding expressions of ref. [9] are (A.19) and (B.1). The calculation of the remaining, $|\alpha\rangle$ partial-wave-projected matrix elements in (35) follows (B.1) of ref. [9].

\section{Summary}

We extended our approach to include the pp Coulomb force into the momentum space $3 \mathrm{~N}$ Faddeev calculations, presented in refs. $[9,10]$ for elastic pd scattering and breakup in case when only pairwise forces are acting, to include also a $3 \mathrm{NF}$ and to treat reactions induced by interaction of electromagnetic probes with the ${ }^{3} \mathrm{He}$ nucleus. It is based on a standard formulation for short-range forces and relies on the screening of the long-range Coulomb interaction. In order to avoid all uncertainties connected with the application of the partial-wave expansion, unsuitable when working with long-range forces, we apply directly the 3 -dimensional pp screened Coulomb $t$-matrix.

For each reaction considered in the present study: elastic pd scattering and breakup, two- and three-body decay of the ${ }^{3} \mathrm{He}$ nucleus induced by real or virtual photons, the resulting coupled set of integral equations in the finite space of $|\alpha\rangle$ channels only, incorporates the contributions of the pp Coulomb interaction from all partial-wave states up to infinity. Adding a 3NF results in a set of Faddeevtype equations with the same structure as in the case when only $2 \mathrm{~N}$ interactions and pp Coulomb force are acting. On top of that for each reaction one new contribution in the leading term and in the kernel appears. These two additional terms have the same form independent if the pp Coulomb force is acting or not.
Solutions of the resulting Faddeev equations in the form of partial-wave-projected matrix elements, together with the additional matrix elements calculated directly with the 3 -dimensional screened Coulomb $t$-matrix, provide transiton amplitudes from which numerous observables can be calculated.

Since in $[9,10]$ the practical feasibility of our formulation has been documented in case of pd elastic scattering and breakup, the presented extension of similar structure will also be feasible and will allow to apply that approach with the complete nuclear Hamiltonian to analyses of numerous data from $3 \mathrm{~N}$ hadronic and electromagnetic reactions.

This work was supported by the Polish 2008-2011 science funds as a research project No. N N202 077435. It was also partially supported by the Helmholtz Association through funds provided to the virtual institute "Spin and strong QCD" (VH-VI-231).

Open Access This article is distributed under the terms of the Creative Commons Attribution Noncommercial License which permits any noncommercial use, distribution, and reproduction in any medium, provided the original author(s) and source are credited.

\section{References}

1. E.O. Alt, W. Sandhas, H. Ziegelmann, Phys. Rev. C 17, 1981 (1978).

2. E.O. Alt, W. Sandhas, in Coulomb Interactions in Nuclear and Atomic Few-Body Collisions, edited by F.S. Levin, D. Micha (Plenum, New York, 1996) p. 1.

3. E.O. Alt, M. Rauh, Phys. Rev. C 49, R2285 (1994).

4. E.O. Alt, A.M. Mukhamedzhanov, M.M. Nishonov, A.I. Sattarov, Phys. Rev. C 65, 064613 (2002).

5. A. Kievsky, M. Viviani, S. Rosati, Phys. Rev. C 52, R15 (1995).

6. A. Deltuva, A.C. Fonseca, P.U. Sauer, Phys. Rev. C 72, 054004 (2005).

7. A. Deltuva, A.C. Fonseca, P.U. Sauer, Phys. Rev. C 71, 054005 (2005).

8. R. Skibiński, J. Golak, H. Witała, Acta Phys. Pol. B 41, 875 (2010).

9. H. Witała, R. Skibiński, J. Golak, W. Glöckle, Eur. Phys. J. A 41, 369 (2009).

10. H. Witała, R. Skibiński, J. Golak, W. Glöckle, Eur. Phys. J. A 41, 385 (2009).

11. W. Glöckle, H. Witała, D. Hüber, H. Kamada, J. Golak, Phys. Rep. 274, 107 (1996).

12. W. Glöckle, The Quantum Mechanical Few-Body Problem (Springer Verlag, 1983).

13. H. Witała, W. Glöckle, H. Kamada, Phys. Rev. C 43, 1619 (1991).

14. A. Deltuva, Phys. Rev. C 80, 064002 (2009).

15. D. Hüber, H. Kamada, H. Witała, W. Glöckle, Acta Phys. Pol. B 28, 1677 (1997).

16. J. Golak, R. Skibiński, H. Witała, W. Glöckle, A. Nogga, H. Kamada, Phys. Rep. 415, 89 (2005). 